\title{
Identifying parasite resistance genes in Solanum tuberosum by RNA-seq
}

\author{
N. Shmakov ${ }^{1 *}$, A. Egorova ${ }^{1}$, A. Glagoleva ${ }^{1,2}$, A. Kochetov ${ }^{1,2}$, D. Afonnikov ${ }^{1,2}$ \\ ${ }^{1}$ Institute of Cytology and Genetics SB RAS, Novosibirsk, Russia \\ ${ }^{2}$ Novosibirsk State University, Novosibirsk, Russia \\ *e-mail:shmakov@bionet.nsc.ru
}

Key words: resistance genes, NBS-LRR, RNA-seq, differential expression, transcriptome assembly

Motivation and Aim: Enhancing resistance of cultivated plants, namely potato Solanum tuberosum, to parasitic organisms is an important but complicated task. It was revealed that nucleotide-binding site-leucine-rich repeat (NBS-LRR) genes compose the largest pathogen resistance gene family [1]. This genes family includes genes potentially responsible for resistance to golden potato cyst nematode (GPCN) Globodera rostochiensis. GPCN is one of the most economically important potato pathogens [2]. Thus, discovery of genes controlling potato resistance to GPCN is an important task. To achieve this goal, we performed RNA-seq analysis of two potato cultivars varying in their GPCN resistance.

Methods and Algorithms: S. tuberosum plants of genotype i-0144786, susceptible to GPCN, and genotype i-0144787, resistant to GPCN, were inoculated with GPCN cysts or treated with water as control group, and total RNA was extracted from plant roots on different time stages. Libraries of paired-end short reads were sequenced using Illumina HiSeq 2500 system. Libraries were filtered from low-quality, low-length reads, singletons, ambiguous reads, and adapter sequences were removed. Libraries were mapped to the reference $S$. tuberosum genome, and search for differential expression was performed. In addition, de novo transcriptome reconstruction was carried out, and, after excluding lowly-presented transcripts, search for differentially expressed transcripts was conducted. Additionally, transcripts were functionally annotated. To achieve this, domain structures were predicted in assembled contigs. Verification of differential expression through qRT-PCR was performed for a selected list of genes.

Results: A number of differentially expressed transcripts were detected both with library mapping analysis and transcriptome de novo assembly. Based on motif homology, NBS-LRR genes were predicted, including a number of de novo assembled transcripts presented in i-0144787 resistant genotype but not in i-0144786 susceptible genotype. Conclusion: In this study we revealed several NBS-LRR genes associated with GPCN resistance

Acknowledgements: Supported by the RSF (16-16-04073). Plants of genotypes i-0144786 and i-0144787 were presented by VIR.

\section{References}

1. Shao Z.Q., Wang B., Chen J.Q. (2016) Tracking ancestral lineages and recent expansions of NBS-LRR genes in angiosperms. Plant Signal Behav. 11(7):e1197470.

2. Kochetov A. et al. (2017) Differential expression of NBS-LRR encoding genes in the root transcriptomes of two Solanum phureja genotypes with contrasting resistance to Globodera rostochiensis. BMC Plant Biology. 17(Suppl. 2):251. DOI 10.1186/s12870-017-1193-1. 